\title{
Efeito da potencialização pós-ativação na performance do nado crawl em nadadores velocistas
}

http://dx.doi.org/10.11606/1807-5509201900040621

\author{
Paolo Veiga SIRIEIRO* \\ Mayro Passos RUSSANO** \\ Humberto Lameira MIRANDA*
}

*Universidade Federal do Rio De Janeiro, Rio de Janeiro, RJ, Brasil. **Universidade Federal Fluminense, Niterói,

RJ, Brasil.

\section{Resumo}

0 objetivo deste estudo foi investigar o efeito da potencialização pós-ativação (PPA) na performance de nadadores velocistas. Participaram dez nadadores homens adultos $(19,6 \pm 2,2$ anos, altura 181,4 $\pm 2,2$ $\mathrm{cm}$; peso $77 \pm 9 \mathrm{~kg}, \mathrm{n}=10$ ), com no mínimo cinco anos de experiência em natação competitiva de alta performance. Todos os nadadores foram avaliados em tiro curto de 15 metros após aquecimento e após 4 repetições máximas no exercício pullover (extensão de ombros na polia alta), ambos na mesma sessão. Os resultados não demonstraram diferença significativa do primeiro para o segundo tiro de 15 metros na performance $(8,49 \pm 0,36$ vs. $8,48 \pm 0,38$ segundos $p=0,83)$ e na velocidade média (VM) $(1,77 \pm 0,07$ vs. $1,77 \pm 0,08 \mathrm{~m} / \mathrm{s} p=0,74)$. A frequência de braçada (FB) $(0,96 \pm 0,07$ vs. 0,97 $\pm 0,07$ hertz $p=0,09)$ e comprimento de braçada (CB) $(1,85 \pm 0,12$ vs. $1,83 \pm 0,11$ metros $p=0,19)$ também não apresentaram diferença estatística. Portanto, a utilização do exercício pullover como método de potencialização pósativação não melhorou a performance de nadadores velocistas em tiro curto.

Palavras-Chave: Nadadores; Desempenho; Potência; Tiro.

\section{Introdução}

Diferentes metodologias são utilizadas na natação competitiva com o objetivo de aumentar a performance dos atletas nadadores. Em um estudo de revisão, ASPENES e KARLSEN ${ }^{1}$ analisaram diferentes formas de treinamento dentro e fora d'água comuns as rotinas de atletas nadadores. Os pesquisadores revisaram 17 artigos, sendo 12 para treinamentos de resistência. Dentre as metodologias encontravam-se: treinamento de força no solo (dry-land), MADsystem (tipo de escada submersa, onde o método envolve os atletas executarem as braçadas puxando os "degraus" dessa escada); treinamento com um tipo de tigela perfurada, usada como sobrecarga de arrasto preso a cintura; e uso de elásticos presos a cintura dos atletas para que pudessem exercer resistência ou assistência para o nado, e todos apresentaram melhora na performance. Além do uso de Biokinetic swim bench (máquina que simula o nado fora d'água) e uso de palmares como forma de sobrecarga no treino e, em ambos, não foi encontrado melhora de performance.
Do ponto de vista de respostas agudas, a potencialização pós-ativação (PPA) vem sendo demonstrada como um fenômeno, ou ferramenta, capaz de aumentar a potência através do estímulo prévio de força. Portanto, um gesto esportivo explosivo poderia ser aumentando quando executado subsequente ao exercício de força máxima ou submáxima². Os mecanismos fisiológicos, apesar de inconclusivos, se baseiam na maior interação actinamiosina na miofibrila, através da fosforilação da cadeia leve de miosina ativada na presença de moléculas de ca liberadas do retículo sarcoplasmático ${ }^{3}$. Sendo assim, a musculatura envolvida assume maior capacidade de produzir força também na forma de potência, e consequentemente performance em atletas ${ }^{2,4-6}$.

Quatro estudos verificaram a influência aguda do PPA na performance de atletas nadadores ${ }^{2,4-6}$. KILLDUFF et al. ${ }^{4}$ comparou o aquecimento tradicional com PPA no agachamento, em 9 nadadores velocistas internacionais (7 homens e 2 mulheres) e percebeu um aumento de pico de força vertical 
e horizontal nas saídas do bloco após PPA, porém a performance dos nadadores analisados durante os 15 metros permaneceu igual em ambos os métodos. Cuenca-Fernández, López-Contreras e ARELLANO ${ }^{5}$ também observaram a performance de saída do bloco em 14 nadadores treinados (10 homens e 4 mulheres), comparando aquecimento tradicional, PPA no agachamento avanço e PPA no agachamento isoinercial bipodal, verificando uma maior eficiência em ambos os métodos de PPA. Porém a PPA agachamento isoinercial bipodal se mostrou mais eficiente em distâncias de 5 metros. Já Hancocck, Sparks e Kullman ${ }^{6}$ compararam a performance de 30 nadadores ( 15 homens e 15 mulheres) em tiro de $100 \mathrm{~m}$ após aquecimento tradicional e aquecimento tradicional + PPA no power rack e, observou o benefício de PPA após verificar aumento significativo de performance no tiro. Todavia, somente Sarramian, Turner e GreENHALGH $^{2}$ analisaram através de tiro na piscina, a influência da PPA utilizando exercício no solo, comparando 18 nadadores de nível nacional (10 homens e 8 mulheres) em quatro diferentes formas de aquecimentos. Foram comparados aquecimento tradicional (AT), aquecimento PPA para membros superiores com "barra" (SPPA), aquecimento PPA para membros inferiores com saltos (IPPA) e aquecimento com PPA combinando membros inferiores e membros superiores (CPPA) e, os autores concluíram que PPA pode ser uma ferramenta para aumentar performance, porém a escolha do exercício "barra" parece não ter sido o estímulo mais apropriado, uma vez que SPPA obteve piora significativa nos tempos dos tiros de 50 metros quando comparado com AT.

Somente Sarramian, Turner e Greenhalgh ${ }^{2}$ verificaram a influência no desempenho de nadadores através da PPA aplicada nos membros superiores utilizando a "barra livre" como exercício de força prévio ao tiro. Uma vez que a maior contribuição propulsiva do nado crawl vem dos braços ${ }^{7}$ e PPA se mostra efetivo quando o movimento biomecânico é mais similar quanto possível do movimento real ${ }^{4,8,9}$, o exercício pullover na polia alta seria do ponto de vista articular (extensão de ombros), o mais semelhante a braçada do nado crawl. Portanto, o presente estudo analisou o efeito da PPA na performance de atletas nadadores em tiros curtos após 4RM do exercício pullover na polia alta. Além do mais, os artigos que avaliaram a performance através de tiros ${ }^{2,4-6,10-12}$ ignoraram variáveis como saída, virada e chegada, sendo que, segundo MaGLISCHO ${ }^{13}$, os tempos de saída, por exemplo, podem representar até $1 / 4$ do tempo total em provas de 25 metros. Sendo assim, o presente artigo procurou minimizar a influência destas variáveis, levando em consideração para fins estatísticos somente os 15 metros centrais do tiro. E assim como no estudo de FARAH et al. ${ }^{14}$, foi utilizado o recurso de filmagem em software editor de vídeo para analisar as variáveis biomecânicas velocidade média $(\mathrm{VM})$, frequência de braçada $(\mathrm{FB})$ e comprimento da braçada (CB).

A hipótese é que após as 4RM no exercício pullover, o alto recrutamento de fibras e unidades motoras ${ }^{3}$ deverá potencializar o tiro de 15 metros, uma vez que as estruturas musculares envolvidas estarão com seu máximo potencial para produzir força na extensão de ombros e, consequentemente, melhorar a performance dos nadadores. Logo, o presente estudo tem como objetivo verificar a influência da PPA na performance de nadadores velocistas.

\section{Método}

\section{Sujeitos}

Para a presente pesquisa dez nadadores adultos foram voluntários (idade 19,6 \pm 2,2 anos, altura $181,4 \pm 2,2 \mathrm{~cm}$; peso $77 \pm 9 \mathrm{~kg}$; massa magra $67,9 \pm$ $7,3 \mathrm{~kg}$; massa gorda $9 \pm 1,7 \mathrm{~kg}$, envergadura $187 \pm 7,9$ $\mathrm{cm}$ ), com mais de 5 anos de experiência em natação competitiva, disputando campeonatos estaduais e nacionais. Como critérios de inclusão adotaramse: não apresentar questionário PAR-Q positivo e não ter histórico de lesões osteomusculares. Como critérios de exclusão adotaram-se: ter utilizado qualquer medicação, suplemento ou recurso ergogênico durante as sessões de testes. $\mathrm{O}$ projeto foi aprovado pelo Comitê de Ética em Pesquisa da Universidade sob o $\mathrm{n}^{\circ}: 003 / 2011$. Todos os indivíduos assinaram o Termo de Consentimento Livre e Esclarecido, conforme a resolução 196/96 do Conselho Nacional de Saúde ${ }^{15}$.

Para medir a estatura e a envergadura foi usada uma trena antropométrica sem trava (TR4010, Sanny, Itu, Brasil), para o peso corporal 
total foi utilizada a balança digital (2920/100, Brinox, Caxias do Sul, Brasil) e para as dobras cutâneas, o Plicômetro científico tradicional (NHN7 49, Cescorf, Porto Alegre, Brasil). Foi utilizado protocolo de Yuhasz de 6 dobras cutâneas (subescapular, tríceps; coxa; supra-ilíaca; abdome e peitoral), $\mathrm{G} \%=(\mathrm{S} 6) \times 0,095+3,64$, para determinar percentual de massa gorda. Os atletas selecionados se encontravam em período de treinamento no meio da temporada (volume semanal entre 30 e $36 \mathrm{~km}$ divididos em 6 sessões, sendo 3 priorizando estímulos aeróbios e 3 priorizando estímulos anaeróbicos lácticos). Todos os nadadores seguiam um programa específico de musculação, realizados três vezes por semana, com aproximadamente uma hora de duração para cada sessão. O exercício extensão de ombros na polia alta (Start, Buick, Rio de Janeiro, Brasil) já fazia parte do programa de treinamento, logo os mesmos já eram familiarizados com a biomecânica do movimento.

\section{Métodos e procedimentos}

\section{Teste de força máxima}

O teste foi realizado na sala de musculação do Parque Aquático Mourisco do Botafogo de Futebol e Regatas localizado na cidade do Rio de Janeiro - Brasil, e o objetivo foi verificar a carga máxima para as $4 \mathrm{RM}$ no exercício pullover (extensão de ombro). $\mathrm{O}$ atletas foram orientados a se posicionarem próximo ao aparelho com leve flexão do tronco, de forma que ao realizarem o movimento conseguissem uma amplitude articular próximo a 180 graus. Todos receberam estímulos verbal de modo a executarem mesma velocidade de movimento, tanto na fase concêntrica como na excêntrica, com a maior eficiência possível. O reteste foi realizado 48 horas depois, seguindo os mesmos procedimentos da primeira sessão.

\section{Coleta de dados}

As coletas de dados foram realizadas na piscina olímpica do Parque Aquático Mourisco do Botafogo de Futebol e Regatas localizado na cidade do Rio de Janeiro - Brasil, e ocorreu 48 horas após o reteste de $4 \mathrm{RM}$ consistindo no teste de performance.

\section{Aquecimento}

Os nadadores executaram o aquecimento em uma piscina de 25 metros, durante 25 minutos, em um total de 1500 metros (600 metros nadando, 300 metros de perna com prancha, 300 metros de braço com boia, $4 \times 12,5$ metros acelerando a cada 45 segundos e, para finalizar, 200 metros nadando em intensidade leve) tudo no nado crawl.

\section{Teste de Performance}

$\mathrm{Na}$ terceira sessão, todos descansaram 5 minutos após o término do aquecimento, para em seguida executar o primeiro tiro de 25 metros. Descansaram 10 minutos depois do primeiro tiro, para então executar o estímulo de força de 4RM no pullover e descansaram mais 3 minutos para logo depois realizar o segundo tiro de 25 metros (FIGURA 1).
$5 \mathrm{MIN}$

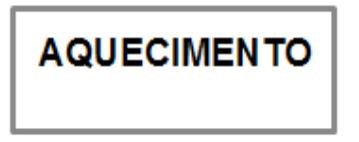

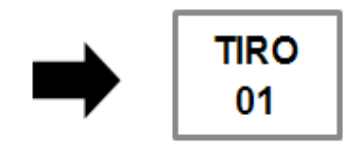

$10 \mathrm{MIN}$

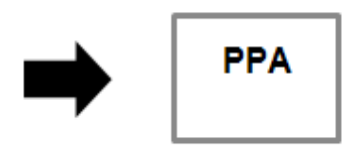

3MIN

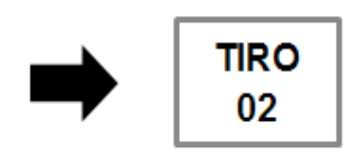

FIGURA 1 - Sequência do protocolo experimental.

\section{Procedimento para o tiro de 25 metros}

Os atletas foram filmados lateralmente, acompanhando o atleta durante todo o percurso dos 25 metros, através de uma câmera filmadora com frequência de 60 quadros por segundo (SDR-S25, Panasonic, Kent, Estados Unidos) e executaram o tiro de dentro d'água, onde foram orientados a executar somente uma pernada submersa (golfinhada) após a impulsão, para então começarem a nadar. Foram excluídos os primeiros 6,25 metros e os últimos 3,75 metros no intuito de descartar a influência da impulsão de borda e desaceleração do nado, respectivamente. Portanto, foi considerado para fins estatísticos somente os 15 metros centrais. 
Procedimento para o teste de performance (tempo de 15 metros)

Através do software editor de vídeos (0.8.15, Kinovea, Paris, França), o tempo de cada nadador foi efetuado através de análise em "câmera lenta" de 20\% da velocidade normal (FIGURA 2). O cronômetro foi disparado quando a cabeça do atleta entrava na marcação dos 6,75 metros da própria piscina e o cronômetro foi parado quando a cabeça do mesmo alcançava a marcação dos últimos 3,75 metros da piscina.

Procedimento para contagem de braçadas, $F B, C B$ e $V M$

A contagem de braçadas do atleta foi efetuada através de análise em "câmera lenta" de $20 \%$ da velocidade normal, permitida pelo software. A contagem iniciou a partir da primeira braçada completa após a cabeça do atleta alcançar a marcação dos 6,25 metros iniciais e terminou com a contagem da última braçada completa no momento em que a cabeça do atleta alcançou a marcação dos 3,75 metros finais.

O que permitiu estipular o número de ciclos de braçadas, no qual a entrada e o retorno da mesma mão na água corresponde um ciclo $(1 \text { ciclo })^{14}$. Logo, a FB expressa em Hertz $(\mathrm{Hz})$, foi calculada pela razão entre 3 ciclos de braçadas ${ }^{16}$ e o tempo levado para realizar o mesmo. A variável VM foi a razão da distância (15 metros) pelo tempo de cada tiro, expresso em metros por segundos $(\mathrm{m} / \mathrm{s})$. E o $\mathrm{CB}$, expresso em metros, foi calculado pela razão da $\mathrm{VM}$ pela $\mathrm{FB}^{14}$.

\section{Procedimento Estatístico}

A estatística descritiva (média e desvio padrão) foi utilizada para descrever as características físicas dos sujeitos. O Método de comparação de médias teste $\mathrm{t}$ para amostras pareadas foi realizada para teste de performance e de velocidade média. Para todo tratamento estatístico foi aceito nível de significância de $5 \%(\mathrm{p}<0,05)$.

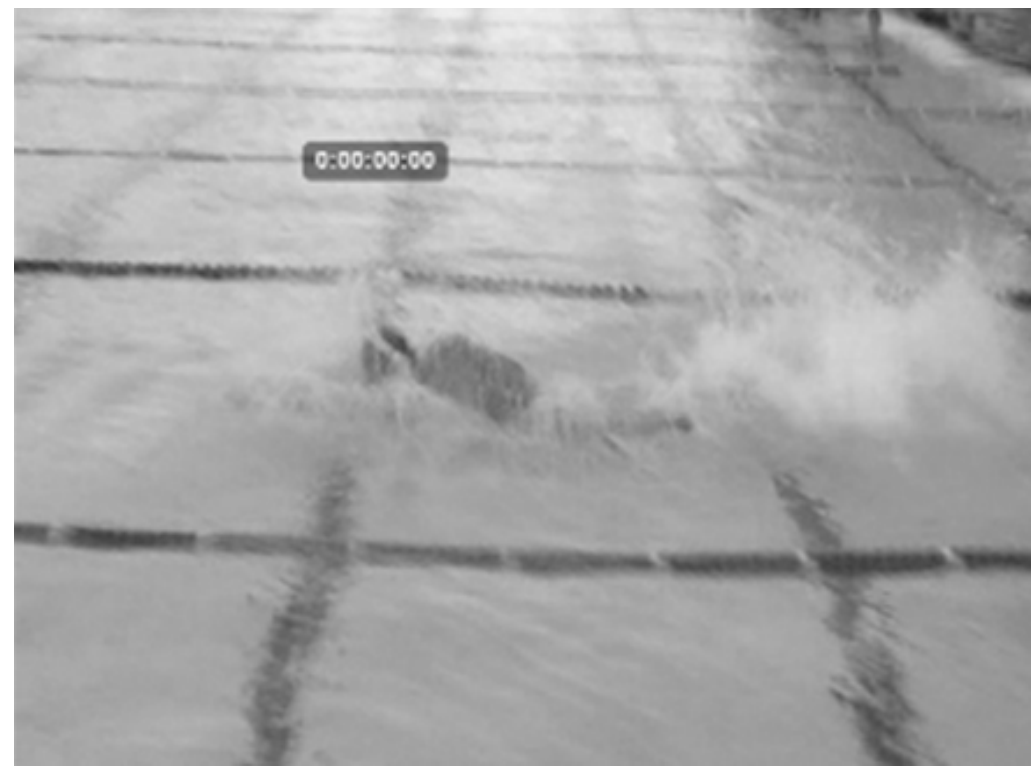

FIGURA 2 -Momento em que a cabeça do nadador atinge a marcação da piscina e o cronômetro inicia a contagem de tempo pelo programa.

\section{Resultados}

Não houve diferença significativa na performance entre o primeiro tiro (T1) e o segundo (T2) $(\mathrm{T} 1=8.49 \pm 0.36$ e $\mathrm{T} 2=8,48 \pm$ $0,38$ segundos $\mathrm{p}=0,83)$ e na velocidade média $(\mathrm{T} 1=1,77 \pm 0,07$ e $\mathrm{T} 2=1,77 \pm 0,07 \mathrm{~m} / \mathrm{s} \mathrm{p}=$
$0,74)$. Frequência de braçada $(0,96 \pm 0,07$ vs. $0,97 \pm 0,07$ hertz $\mathrm{p}=0,09)$ e comprimento de braçada $(\mathrm{CB})(1,85 \pm 0,12$ vs. $1,83 \pm 0,11$ metros $\mathrm{p}=0,19)$ também não apresentaram diferenças (TABELA 1). 
TABELA 1 -Média e desvio padrão de performance, velocidade média, Frequência de braçada e comprimento de braçada.

\begin{tabular}{cccc}
\hline & Tiro $1(n=10)$ & Tiro 2 $(n=10)$ & P \\
\hline Performance $(\mathrm{s})$ & $8,49 \pm 0,36$ & $8,48 \pm 0,38$ & 0,83 \\
Velocidade Média $(\mathrm{m} / \mathrm{s})$ & $1,77 \pm 0,07$ & $1,77 \pm 0,07$ & 0,74 \\
Frequência de Braçada $(\mathrm{Hz})$ & $0,96 \pm 0,07$ & $0,97 \pm 0,07$ & 0,09 \\
Comprimento de Braçada $(\mathrm{m})$ & $1,85 \pm 0,12$ & $1,83 \pm 0,11$ & 0,19 \\
\hline
\end{tabular}

Os dados são apresentados como média e desvio padrão. $\alpha>0,05$ (p-valor).

\section{Discussão}

Após a realização de um exercício resistido, a musculatura se encontra em distintas situaçóes, fadiga e potencialização. O equilíbrio entre ambos é a chave para o subsequente movimento explosivo ou performance. Todavia, muitos são os fatores que influenciam na PPA, como; atividade, tipo de contração, nível de treinamento dos indivíduos, volume, intensidade, gênero e intervalo de descanso ${ }^{17}$. Portanto, é preciso levar em consideração as peculiaridades do desporto e, sabendo que no nado crawl a maior força propulsiva vem dos braços ${ }^{7}$, a utilização de um exercício para membros superiores que estimule PPA pode ser uma estratégia viável. Se por uma lado a literatura é escassa em pesquisas de PPA em nadadores ${ }^{2,4-6}$, por outro, SARRAmian, TuRnER e Greenhalgh ${ }^{2}$ foram os únicos a avaliarem a potencialização do membros superiores através do exercício barra, mas não encontrou diferenças na performance. Segundo Maglischo ${ }^{13}$ a braçada no nado crawl possui cinco fases distintas, sendo a fase inicial denominada entrada e deslize e a fase final da braçada denominada varredura para cima e recuperação, totalizando uma amplitude articular de extensão de ombros de $180^{\circ}$ nas fases propulsivas. Baseado nessas informaçóes, o exercício pullover mostrou-se do ponto de vista articular e biomecânico para exercício no solo, o mais similar para o movimento e, consequentemente, o mais similar para musculatura agonista envolvida ${ }^{18}$. Além do mais Sarramian, Turner e Greenhalgh ${ }^{2}$ concluíram que a escolha do exercício barra livre parece não ter sido a mais adequada, questionando a importância da influência da baixa ativação do tríceps no exercício barra, uma vez que uma das fases mais propulsivas do nado crawl é a varredura para cima $^{13}$, e nela, a participaçáo do tríceps é de fundamental importância ${ }^{19}$, por se tratar de um movimento combinado de extensão de ombros e extensão de cotovelos. Haja visto que na literatura não existe nenhum estudo que avalie ativação do tríceps no exercício pullover em comparação com a barra livre, Campos e Silva ${ }^{20}$ ao compararem o exercício pullover com o supino horizontal verificaram maior atividade eletromiográfica do tríceps braquial em relação a sua ativação no supino horizontal ( $\mathrm{p}<0.014, \mathrm{~d}=0.268$, small effect size), o que evidencia sua forte contribuição neste exercício. Logo a escolha do pullover no presente estudo, se apresenta como uma possibilidade de exercício capaz de produzir maior ativação de tríceps, e assim, contribuir de forma eficaz, também, na varredura para cima.

Entretanto, o presente estudo não encontrou diferenças significativas entre o primeiro e o segundo tiro realizado após PPA no pullover. Uma possível explicação seria o fato que, segundo MAGLISCHO ${ }^{13}$, apesar de muito comum os treinamentos de força no solo, a transferência dessa força para a performance dos nadadores em tiros curtos não ocorre completamente porque tão importante quanto aumentar os ganhos de força nas fibras musculares envolvidas é a capacidade do sistema nervoso central em recrutar essas mesmas fibras em uma sequência correta para realização, nesse caso específico, do movimento no nado crawl. O que leva a uma maior consideração sobre a influência da variável técnica de nado dentro da especificidade do desporto. Ao executar o movimento em um aparelho, como o pullover na polia alta, toda força aplicada pelos braços na barra de apoio será proporcionalmente transformada em deslocamento da mesma, por outro lado, no meio líquido, o deslocamento do atleta na água depende da aplicação de força em uma base não sólida e instável, que segundo MAGLISCHO ${ }^{13}$, implica em duas grandes desvantagens: a primeira é que ao se deslocar na água, o meio líquido oferece menos 
resistência as forças propulsivas. A segunda, é que devido a sua densidade, a água oferecer maior resistência para os nadadores se deslocarem. A partir daí fica claro a importância e a real existência de um padrão de técnicas específicas durante cada estilo, para que o nadador possa sempre vencer a resistência e deslocar o máximo de massa de água a favor de sua propulsão, a qual tal especificidade técnica é acompanhada de uma sequência de recrutamento de fibras muito específico para aquele gesto motor do desporto. Portanto, por mais similar que o movimento biomecânico do pullover pareça ser com a braçada, ainda assim não foi específico o suficiente para desencadear a PPA.

Logo após estímulo de força, o músculo permanece igualmente em um estado de potencialização e fadiga ${ }^{3,21}$. Porém, a recuperação da fadiga acontece de forma mais rápida que a condição de potencialização criando uma "janela de oportunidade" onde essa vantagem muscular compreende intervalos de recuperação de 4-12 minutos ${ }^{3,6,21}$. Os estudos que verificaram a influência da PPA na performance de nadadores utilizaram intervalos de recuperação de pelo menos 4 minutos de intervalo ${ }^{2-6}$ corroborando com a teoria da "janela de oportunidade". Todavia, o presente estudo utilizou o intervalo de 3 minutos entre o estímulo de força e o tiro de 25 metros, o que, também, pode ter influenciado diretamente nos resultados de performance, não sendo talvez, o suficiente para recuperação da fadiga. Por outro lado, Tiluin e Bishop ${ }^{3}$ citam a presença de duas “janelas de oportunidade”, sendo uma delas a já citada, e a outra, imediatamente após ao estímulo de força em uma condição de baixo volume de estímulo de força onde PPA é dominante em relação a fadiga, o que pode ser a circunstância do presente estudo que utilizou apenas uma série de 4RM no exercício pullover. Além disso, WiLsON et al. ${ }^{17}$ em uma metanálise, mostrou através do efeito do tamanho que quanto maior o nível de treinamento dos indivíduos, maior sua resistência a fadiga e logo, menor seria a necessidade do tempo de recuperação, observando uma janela de oportunidade para atletas de 3-7 minutos, corroborando com o intervalo de recuperação do presente estudo. Tillin e BishOP ${ }^{3}$ sugerem que a relação PAP X Fadiga é contraditória e muitos fatores como volume, intensidade do estímulo de força, tipo de contração, características dos sujeito (força muscular, distribuição do tipo de fibras e treinabilidade) e o tipo de atividade realizada após estímulo de força influenciam em uma maior ou menor necessidade de recuperação da fadiga, tornando essa relação multi-fatorial e de evidências não conclusivas.

Em estudo de revisão com métodos de treinamento de força para nadadores, Pires, Figueira Júnior e MirANDA $^{22}$ constataram que $73,3 \%$ das pesquisas avaliavam performance através de tiros curtos (10-50 metros), seguidos por tiros de 100 metros $(13,3 \%)$ e 400 metros (13,3\%). Todavia, apesar de haver um determinado padrão de metragem para avaliar performance em nadadores, não existe um protocolo específico que tente minimizar o efeito de algumas variáveis que interferem diretamente no tempo de performance e que na maioria da vezes não são objetivo do estudo, como é o caso das saídas, viradas e chegadas. Os tempos de saída podem representar até $1 / 4$ do tempo total em provas de 25 , já as viradas gastam cerca $20-38 \%$ do tempo em provas de piscina curta e uma chegada pouco eficiente pode representar 0,10 segundos, ou mais, no tempo do nadador ${ }^{13}$. Sendo assim, o presente artigo procurou minimizar os efeitos dessas variáveis através do tiro de 25 metros em piscina com mesmo tamanho - excluindo assim a necessidade de viradas, e considerando para fins estatísticos de performance somente os 15 metros centrais. Ao colocar o atleta para fazer o tiro de dentro d'água e excluir os 6,25 primeiros metros, o que se espera é minimizar a influência do tempo de reação no bloco de partida e da aceleração do nado pelo impulso na borda da piscina, respectivamente, descartando assim a variável saída. E a exclusão dos 3,75 metros finais procurou minimizar a influência da desaceleração do nado e da variável chegada. Porém, como a diferença de 1 centésimo entre o tiro após aquecimento e o tiro após o estímulo de força não foi significativo, é possível que a curta distância (15 metros) não seja suficiente para averiguar influência na performance, uma vez que os atletas são treinados com mais de 5 anos de experiência em natação competitiva e possuem alta capacidade de reproduzir um padrão de tempo em tiros curtos durante as várias fases do treinamento.

Apesar da tentativa de ser o mais fiel o quanto possível para elucidar essa transferência de força pela biomecânica do movimento articular e dos músculos solicitados, uma das limitações do estudo parece ter sido a metragem de 15 metros em tiro curto utilizada para avaliar a performance dos atletas, não sendo suficiente para verificar resultados significativos nos efeitos da PPA. Reconhecemos como limitação também, o fato dos tiros serem realizados seguidos, em sessão única, já que não houve uma situação controle para saber se o primeiro tiro afetou o 
segundo, tanto do ponto de vista negativo onde a fadiga poderia estar interferindo no segundo tiro, quanto positiva se não teria retardado a perda de aquecimento e consequentemente, de desempenho, pelo intervalo de 13 minutos entre os tiros. Novas pesquisas devem ser realizadas e com diferentes exercícios, intensidades, volume e intervalos de recuperação, para investigar a possibilidade da transferência de força em forma de potência com exercícios de membros superiores no solo e dentro do próprio meio líquido, e até mesmo comparar a diferença de performance entre eles. Além de buscar um protocolo ou método que minimize a influência de variáveis que influenciam diretamente no resultado da análise da performance em tiros curtos como as saídas, viradas e chegadas.

Se tratando de exercícios similares quanto ao gesto mecânico e a forma de aplicação da força, é possível potencializar padrōes motores explosivos com prévio estímulo de força. Porém, a natação lida com peculiaridades nos padróes propulsivos de aplicação de força, no qual, a técnica de nado associado ao sequencial recrutamento de fibras pelo sistema nervoso central faz com que determinadas hipóteses criadas em modalidades no solo possam não ser direta ou completa quando levadas do solo para o meio líquido. Portanto, a utilização do exercício pullover como método de potencialização pós-ativação não melhorou a performance de nadadores velocistas em tiro curto.

\begin{abstract}
Effect of postactivation potentiation on front crawl performance in sprint swimmers

The purpose of this study was to investigate the effect of post-activation potentiation (PAP) in the performance of sprinters swimmers. Ten men swimmers $(19.60 \pm 2.23$ years, height $181.45 \pm 2.23 \mathrm{~cm}$, weight $77 \pm 9.03 \mathrm{~kg}, \mathrm{n}=10$ ), with at least five years of experience in high performance competitive swimming participated in this study. All swimmers were evaluated in 15 meters short sprint and short sprint after 4 repetition maximum performance in the pullover exercise (shoulder extension in the high pulley), both in the same session. The results showed no significant difference from the first to the second sprint from 15 meters in performance ( $8.49 \pm 0.36$ vs. $8.48 \pm 0.38$ seconds on the $2 n d ; p=0.83$ ) and average speed $(1.77 \pm 0.07$ vs. $1.77 \pm 0.08 \mathrm{~m} / \mathrm{s} ; p=0.74)$. The stroke rate $(0,96 \pm 0,07$ vs. $0,97 \pm 0,07$ hertz $p=0,09)$ and stroke length $(1,85 \pm 0,12$ vs. $1,83 \pm 0,11$ meters $p=0,19)$ did not show statistical difference. Therefore, the use of the pullover exercise as post-activation potentiation method does not improved performance swimmers sprinters in acute state.
\end{abstract}

KeYwoRds: Swimmers; Performance; Power Output; Sprint.

\title{
Referências
}

1. Aspenes ST, Karlsen T. Exercise-training intervention studies in competitive swimming. Sports Med. 2012;42(6):527-43.

2. Sarramian VG, Turner AN, Greenhalgh AK. Effect of postactivation potentiation on fifty-meter freestyle in national swimmers. J Strength Cond Res. 2015;29(4):1003-9.

3. Tillin NA, Bishop D. Factors modulating post-activation potentiation and its effect on performance of subsequent explosive activities. Sports Med. 2009;39(2):147-66.

4. Kilduff LP, Cunningham DJ, Owen NJ, West DJ, Bracken RM, Cook CJ. Effect of postactivation potentiation on swimming starts in international sprint swimmers. J Strength Cond Res. 2011;25(9):2418-23.

5. Cuenca-Fernández F, López-Contreras G, Arellano R. Effect on swimming start performance of two types of activation protocols: lunge and YoYo squat. J Strength Cond Res. 2015;29(3):647-55.

6. Hancock AP, Sparks KE, Kullman EL. Postactivation potentiation enhances swim performance in collegiate swimmers. J Strength Cond Res. 2015;29(4):912-7.

7. Deschodt VJ, Arsac LM, Rouard AH. Relative contribution of arms and legs in humans to propulsion in 25-m sprint 
front-crawl swimming. Eur J Appl Physiol Occup Physiol. 1999;80(3):192-9.

8. Esformes JI, Keenan M, Moody J, Bampouras TM. Effect of different types of conditioning contraction on upper body postactivation potentiation. J Strength Cond Res. 2011;25(1):143-8.

9. Samson M, Button DC, Chaouachi A, Behm DG. Effects of dynamic and static stretching within general and activity specific warm-up protocols. J Sports Sci Med. 2012;11(2):279-85.

10. Girold S, Maurin D, Dugué B, Chatard J-C, Millet G. Effects of dry-land vs. resisted- and assisted-sprint exercises on swimming sprint performances. J Strength Cond Res. 2007;21(2):599-605.

11. Trappe SW, Pearson DR. Effects of Weight Assisted Dry-Land Strength Training on Swimming Performance. J Strength Cond Res. 1994;8(4):209-13.

12. Aspenes S, Kjendlie P-L, Hoff J, Helgerud J. Combined strength and endurance training in competitive swimmers. J Sports Sci Med. 2009;8(3):357-65.

13. Maglischo EW. Nadando o mais rápido possível. 3a ed. Barueri: Manole; 2010.

14. Farah BQ, Silva WCM, Santos MA, Melo WV de C. Análise descritiva do desempenho em uma prova de $100 \mathrm{~m}$ nado livre feminino baseada em variáveis biomecânicas. Rev Bras Educ Fís Esporte. 2010;24(4):463-9.

15. Brasil. Conselho Nacional de Saúde. Aprovar as seguintes diretrizes e normas regulamentadoras de pesquisas envolvendo seres humanos. Resolução n.196, 10 outubro 1996.

16. Toubekis AG, Adam GV, Douda HT, Antoniou PD, Douroundos II, Tokmakidis SP. Repeated sprint swimming performance after low- or high-intensity active and passive recoveries. J Strength Cond Res. 2011;25(1):109-116.

17. Wilson JM, Duncan NM, Marin PJ, Brown LE, Loenneke JP, Wilson SMC, et al. Meta-analysis of postactivation potentiation and power: effects of conditioning activity, volume, gender, rest periods, and training status. J Strength Cond Res. 2013;27(3):854-9.

18. Hall SJ. Biomecânica Básica. 5a edição. Barueri: Guanabara; 2009.

19. Figueiredo P, Sanders R, Gorski T, Vilas-Boas JP, Fernandes RJ. Kinematic and electromyographic changes during 200 $\mathrm{m}$ front crawl at race pace. Int J Sports Med. 2013;34(1):49-55.

20. Campos YAC, Silva SF. Comparison of electromyographic activity during the bench press and barbell pulloverexercises. Motriz: rev educ fís. 2014;20(2):200-205.

21. Hodgson M, Docherty D, Robbins D. Post-activation potentiation: underlying physiology and implications for motor performance. Sports Med. 2005;35(7):585-95.

22. Pires GP, Figueira Júnior A, Miranda MLJ. Treinamento de Força para Nadadores Competitivos: Uma Revisão Sistemática Acerca dos Métodos e dos Resultados na Força Muscular e Desempenho na Natação. R Bras Ci e Mov. 2014;22(2):148-62.

ENDEREÇO DE CORRESPONDÊNCIA:

Paolo Veiga Sirieiro

Rua Senador Vergueiro, 148, ap 707 - Flamengo

Rio de Janeiro - RJ - BRASIL

CEP: 22230-001

E-mail: paolosirieiro@ufrj.br; paolo_cf@hotmail.com
Submetido: $18 / 05 / 2016$

$1^{\text {a }}$ revisão: $25 / 08 / 2016$

$2^{\text {a }}$ revisão: $03 / 12 / 2016$

$3^{\text {a }}$ revisão: 29/03/2017

Aceito: 08/05/2017 